\title{
Desempeño de cartas de control estadístico con límites bilaterales de probabilidad para monitorear procesos Weibull en mantenimiento
}

\section{Performance of Statistical Control Charts with Bilateral Limits of Probability to Monitor Processes Weibull in Maintenance}

\author{
Quintana Alicia Esther \\ Departamento de Matemática \\ Universidad Nacional del Sur, República de Argentina \\ Correo:quintana@uns.edu.ar \\ Pisani María Virginia \\ Departamento de Matemática \\ Universidad Nacional del Sur, República de Argentina \\ Correo:mvpisani@uns.edu.ar
}

\author{
Casal Ricardo Néstor \\ Departmento de Ingeniería \\ Universidad Nacional del Sur, República de Argentina \\ Correo:rcasal@uns.edu.ar
}

Información del artículo: recibido: noviembre de 2013, aceptado: marzo de 2014

\begin{abstract}
Resumen
Una manufactura con óptimos estándares de calidad está apuntalada por la alta confiabilidad de sus equipos y sistemas, entre otros pilares esenciales. Ingeniería de mantenimiento tiene la responsabilidad de planear el control y mejoramiento continuo de su equipamiento crítico mediante algún enfoque, como Six Sigma. Este se nutre de numerosas herramientas estadísticas destacando, entre ellas, a las cartas de control estadístico de procesos. Si bien sus primeras aplicaciones fueron en producción, otros diseños surgieron para adaptarse a nuevas necesidades como el monitoreo de los equipos y sistemas en el hábitat fabril. El tiempo entre fallas suele ajustarse a un modelo Exponencial o Weibull. Las cartas t y $\mathrm{t}$ ajustada, con límites probabilísticos de control, son alternativas aptas para monitorear el tiempo medio entre fallas. Desafortunadamente, es difícil encontrar publicaciones de ellas aplicadas a modelos Weibull, muy útiles en contextos como mantenimiento. Además, la literatura limita el estudio de su desempeño al análisis de la métrica estándar: longitud de corrida promedio, dando así una visión parcial. El objetivo de este trabajo es explorar el desempeño de las cartas t y las cartas $t$ ajustada mediante el uso de tres métricas, dos no convencionales. Para ello, incorpora el concepto de variabilidad lateral, en sus formas variabilidad derecha e izquierda. Mayores precisiones del comportamiento de estas cartas permitieron entender las condiciones bajo las cuales son convenientes: si el objetivo principal del monitoreo recae en detectar deterioros, se recomienda la carta $t$ con ajuste. En cambio, cuando la prioridad es detectar mejoras, la carta t sin ajuste es la mejor opción. No obstante, la velocidad de respuesta de ambas cartas es muy variable de corrida en corrida.
\end{abstract}

\section{Descriptores:}

- mantenimiento

- confiabilidad

- Weibull

- cartas de control con límites de probabilidad

- carta t

- carta t-ajustada

- LCP insesgada

- variabilidad lateral 


\begin{abstract}
Manufacturing with optimal quality standards is underpinned to the high reliability of its equipment and systems, among other essential pillars. Maintenance Engineering is responsible for planning control and continuous improvement of its critical equipment by any approach, such as Six Sigma. This is nourished by numerous statistical tools highlighting, among them, statistical process control charts. While their first applications were in production, other designs have emerged to adapt to new needs as monitoring equipment and systems in the manufacturing environment. The time between failures usually fits an exponential or Weibull mod$e l$. The $t$ chart and adjusted $t$ chart, with probabilistic control limits, are suitable alternatives to monitor the mean time between failures. Unfortunately, it is difficult to find publications of them applied to the models Weibull, very useful in contexts such as maintenance. In addition, literature limits the study of their performance to the analysis of the standard metric average run length, thus giving a partial view. The aim of this paper is to explore the performance of the tchart and adjusted tchart using three metrics, two unconventional. To do this, it incorporates the concept of lateral variability, in their forms left and right variability. Major precisions of the behavior of these charts allow to understand the conditions under which are suitable: if the main objective of monitoring lies in detecting deterioration, the $t$ chart with adjustment is recommended. On the other hand, when the priority is to detect improvements, the tchart without adjustment is the best choice. However, the response speed of both charts is very variable from run to run.
\end{abstract}

\section{Introducción}

En el contexto mundial competitivo actual, el control y mejoramiento continuo de los procesos críticos de una empresa mediante un programa sistemático como por ejemplo, Six Sigma, se reconoce no solo como una necesidad, sino también como una estrategia de negocios, una ventaja competitiva (Gutiérrez y de la Vara, 2009). Ingeniería del mantenimiento no escapa a esta realidad; por el contrario, desempeña una función cada vez más comprometida dentro de las empresas. Esto se ve reflejado en los diversos cambios que sus prácticas diarias experimentan desde hace años.

La obtención de una manufactura con estándares de calidad óptimos está respaldada esencialmente por la alta confiabilidad de su equipamiento y sistemas, entendiéndose esta como su capacidad para suministrar largos períodos de desempeño satisfactorio sin fallas. Ingeniería de mantenimiento tiene entonces la responsabilidad clave de elaborar y ejecutar un plan de control y mejoramiento continuo de la confiabilidad de sus activos críticos, identificando y tratando adecuadamente deterioros o aspectos posibles de mejora.

Si bien las primeras aplicaciones de la metodología Six Sigma surgieron en manufactura, se han extendido a otras áreas y continúa creciendo. Un programa Six Sigma comprende una serie de etapas bien definidas: definir, medir, analizar, mejorar y controlar. Cada una de ellas se nutre de una gran diversidad de herramien- tas estadísticas. La última se apoya en el control estadístico de procesos (CEP) destacando, como su principal herramienta, las cartas de control.

\section{Cartas de control}

Son métodos gráficos para monitorear y diagnosticar el desempeño de un proceso en el tiempo, detectando posibles corrimientos de magnitud $D$ en los valores nominales de sus principales parámetros; por ejemplo, en la media o desviación estándar de una variable descriptiva de su desempeño (Surucu y Sazak, 2009; Ryan, 2011). Las cartas bilaterales tienen un doble rol: permiten detectar corrimientos por abajo $(D<0)$ y por arriba $(D>0)$ del valor nominal del parámetro, lo que señala deterioros y mejoras en el desempeño. Las cartas bilaterales son entonces claves en todo programa de mejoramiento continuo y, en particular, del pensamiento Six Sigma. Lecturas periódicas del desempeño del proceso se vuelcan a un estadístico de control cuya estructura matemática depende del tipo de carta. Sus valores, representados en la gráfica mediante puntos, se comparan con dos líneas horizontales: los límites de control superior $(L S)$, para detectar corrimientos $D>0$, e inferior $(L I)$, para corrimientos $D<0$.

Con reglas de decisión preestablecidas, si el parámetro ha sufrido cambios $(D \neq 0)$ la carta emite una señal de alerta y se concluye que el proceso está fuera de control con una consecuente toma de acciones en fun- 
ción del tipo de cambio, perjudicial o beneficioso. De lo contrario $(D=0)$, se concluye que el proceso está bajo control en cuyo caso sigue su curso normal. La regla de decisión estándar para cartas de control bilaterales indica que un solo punto fuera de alguno de los límites es señal de alerta y debe actuarse en consecuencia. Aún cuando $D=0$, la carta puede emitir una señal de falsa alarma. Para minimizar su riesgo, se fija de antemano la probabilidad $p_{0}$ definida, bajo la regla de decisión estándar para cartas bilaterales por:

$p_{0}=$ riesgo tolerable de emisión de una señal de falsa alarma

$=P($ un punto arriba de LS si $D=0)+P$ (un punto abajo de LI si $D=0$ )

A partir de $p_{0}$ y los principios estadísticos se establecen los límites de control. La teoría general de las cartas de control nace de la mano de Shewhart en la segunda década del siglo pasado con el objeto de monitorear procesos de manufactura que pueden modelarse con una distribución de probabilidad normal.

Con el tiempo y el avance tecnológico, otros diseños emergieron para cubrir nuevas necesidades y contextos de uso más allá de la producción, por ejemplo en mantenimiento, para monitorear la confiabilidad de los equipos y sistemas en su hábitat fabril. La variable de monitoreo más usual es TBE (Time Between Events). Si bien este término se usa en un contexto más amplio, en confiabilidad representa el tiempo entre fallas consecutivas del equipo o sistema. Desde el punto de vista estadístico, la inestabilidad del activo se manifiesta mediante una distorsión de la variable $T B E$ en su media, variabilidad o distribución, situaciones que pueden ser detectadas con una carta de control. Es un excelente recurso para combinar con actividades de mantenimiento preventivo permitiendo reducir costos y tiempo (Linderman et al., 2005; Zhao et al., 2012).

Si las fallas del proceso se pueden describir por un proceso de Poisson, lo que implica que la tasa de fallas es constante, la distribución exponencial es adecuada para modelar datos TBE (Xie et al., 2002; Zhao et al., 2012). Sin embargo, las maquinarias sufren procesos de envejecimiento o desgaste con lo cual la tasa de fallas crece con el tiempo. En estos casos, los modelos de probabilidad Weibull son los más indicados. Su versatilidad le ha permitido ganar un lugar protagónico en varios contextos de uso: mantenimiento, producción y servicios. Así lo evidencia la literatura y el ámbito empresarial que, desde hace décadas, muestran interés por ellos y no cesa en la actualidad (Lai et al., 2006).
Diseños de cartas de control

En presencia de procesos asimétricos como, por ejemplo, los que pueden modelarse por la distribución de probabilidad de Weibull, las cartas de Shewhart no se comportan bien. Causan, entre otras desventajas, un incremento sustancial en la probabilidad de emisión de una señal de falsa alarma y la posibilidad de un límite de control inferior inexistente con lo cual, la detección de mejoramientos del proceso, clave en un programa Six Sigma, no es factible. Numerosos diseños surgen para corregir estos y otros problemas. Sin pretender hacer una lista exhaustiva, se citan las cartas: CUSUM, EWMA, no paramétricas, basadas en el método de las varianzas ponderadas y con límites de probabilidad.

Los esquemas CUSUM y EWMA son muy veloces para detectar pequeños cambios en los parámetros de interés del proceso. Si bien su diseño surgió para monitorear procesos normales, con el tiempo se extendió a modelos asimétricos (Hawkins y Olwell, 1998; Castagliola et al., 2006; Ryan, 2011). Las cartas no paramétricas tienen la ventaja de que no se basan en una premisa acerca del modelo de probabilidad que rige a la variable de monitoreo. Los trabajos de Bakir (2004) y Chakraborti y van de Wiel (2008) se basan en conocidos tests no paramétricos. Alemi (2004) propuso una sencilla y atractiva carta no paramétrica con base en el gráfico de caja (box plot). Un interesante trabajo para monitorear tiempos de falla puede encontrarse en Li et al. (2012). Las cartas no paramétricas son insensibles ante la presencia de valores atípicos (outliers) como los que pueden producirse con una variable de monitoreo que se modela con una distribución de probabilidad asimétrica como Weibull, por lo que varios autores mostraron interés en cartas con límites de control construidos con base en el método de la varianza ponderada, entre ellos Khoo et al. (2009) y Castagliola y Khoo (2009).

El interés de este trabajo se enfoca en las cartas con límites bilaterales de probabilidad. Conservan los principios de las cartas de Shewhart, pero corrigen sus problemas ante procesos asimétricos, su diseño es más amigable que el de otras cartas mencionadas. Una interesante variedad de diseños, refinamientos y aplicaciones de cartas con límites de probabilidad emergen a fines de la década de los 80 y en forma ininterrumpida hasta nuestros días como respuesta a la necesidad de monitorear procesos de alto rendimiento o alta calidad (high-quality o high-yield processes), que se caracterizan por tener una tasa de fallas muy baja, propias de un ambiente Six Sigma. Entre sus diseños, aptas para ambientes de producción automática, se cuenta con las cartas CCC (Cumulative Count Control Chart) y su exten- 
sión CCC-r. También, sus equivalentes continuas: las cartas CQC (Cumulative Quantity Control Chart) y CQC-r (Xie y Goh, 2006).

Basadas en las cartas CQC y su extensión, surgen en la última década otros esquemas con límites de probabilidad para usarse en contextos más allá de la producción. Se trata de las cartas $\mathrm{t}$ ( $\mathrm{t}$ chart) y su extensión a cartas $t_{r}\left(t_{r}\right.$ chart) introducidas por Xie et al. (2002) con el objeto de monitorear la confiabilidad de los equipos y sistemas de una empresa a través de la media de la variable TBE y suponiendo que estos tiempos pueden modelarse con la distribución de probabilidad exponencial o Weibull. En el caso de la carta t, la gráfica registra directamente las observaciones TBE. En cambio, en la carta $t_{r^{\prime}}$ se maneja el "tiempo total transcurrido hasta la obtención de $r$ fallas". Estas cartas no son exclusivas del área de mantenimiento, pueden utilizarse también en servicios (Santiago y Smith, 2013). En la literatura, las cartas CCC, CQC, t y sus extensiones suelen agruparse dentro de un grupo más general de cartas llamado cartas TBE (TBE Charts) (Liu et al., 2006).

\section{Desempeño de las cartas de control}

A la hora de seleccionar una carta, es imprescindible examinar su desempeño en términos de su velocidad de respuesta ante un corrimento $D$ en el parámetro. La métrica usual es la longitud de corrida promedio $(L C P)$, representa la media de la variable longitud de corrida $(L C)$ definida por:

$L C=$ número de puntos a graficar en la carta desde que se produce un corrimiento $D \neq 0$ en el parámetro hasta que la carta lo detecta

$L C P$ es una función que depende del corrimiento $D$. Cuando $D=0, L C P$ toma aquí la notación $L F A P$ para representar la longitud de falsa alarma promedio, la media de la variable longitud de falsa alarma (LFA). La carta tendrá un mejor desempeño cuanto mayor sea su $L F A P$ y menor su $L C P$ para un rango de valores $\mathrm{D}$ de interés.

Desafortunadamente, la variable $L C$ está afectada por una alta variabilidad (Ryan, 2011; Govindaraju y Lai, 2004) por lo que el mero uso de $L C P$ es insuficiente para mostrar el comportamiento real de la carta, solo brinda una visión parcial. Sin embargo, en la literatura, numerosos estudios de desempeño aún se focalizan, exclusivamente en el análisis de $L C P$. Afortunadamente, el número de publicaciones con análisis más completos está creciendo. Lo más usual es complementar la $L C P$ con otras medidas de resumen de uso corriente en la disciplina estadística (Luceño y Puig, 2000; Tang y Cheong, 2004; Zhang et al., 2006; Chakraborti, 2007).

En otros enfoques, una medida alternativa a $L C P$ apta para corridas cortas de producción fue propuesta por Govindaraju (2005). Gráficos amigables introducidos por Radson y Boyd (2005) permiten comparar rápidamente varios diseños a la vez. Govindaraju y Lai (2004) redefinen la $L C$ modificando la regla de decisión estándar. En cartas donde el tiempo para graficar dos puntos consecutivos varía de un par a otro como en las cartas CCC, CQC, t y sus extensiones, si el factor tiempo es de interés, la métrica ATS (Average Time to Signal) es adecuada; o, en sus formas particulares, ANI (Average Number Inspected) para las cartas CCC y CCC-r y AQI (Average Quantity Inspected) para la carta CQC (Sharma et al., 2006; Liu et al., 2006; Lai y Govindaraju, 2008; Lai et al., 2001).

Quintana et al. (2009) y (2011) presentan un método no convencional al demostrar que las varianzas de $L C$ y $L F A$ pueden descomponerse en dos varianzas laterales, a izquierda y derecha de $L C P$ y $L F A P$, respectivamente. A partir de este resultado proponen, para cada estado del proceso $(D=0$ y $D \neq 0)$, una terna diferente de medidas de resumen, una convencional y dos no. Para $D=0$, la terna está integrada por: $L C P$, la variabilidad izquierda y la proporción de corridas tempranas (con $L C$ inferior a $L C P$ ). En cambio, para $D \neq 0$ : $L C P$, la variabilidad derecha y la proporción de corridas tardías (con $L C$ superior a $L C P$ ). La información combinada de las 3 medidas permite caracterizar, para cada estado del proceso, el desempeño real de la carta de forma más completa y precisa.

\section{Cartas con límites de probabilidad}

Desafortunadamente, las cartas con límites de probabilidad presentan una desventaja. Es de sentido común pretender de una carta que su $L C P$ sea inferior a $L F A P$ para todo corrimiento $D \neq 0$. En términos gráficos, significa que se pretende que la curva $L C P$ tenga su máximo en $D=0$. Se dice entonces que la carta presenta un desempeño con $L C P$ no sesgada ( $A R L$ unbiased) (Ryan, 2011). Esta propiedad no se satisface en las cartas con límites de probabilidad. No obstante, como solución, es posible ajustar sus límites. Una alternativa es asignar valores desiguales a las probabilidades en (1) (Chen y Cheng, 2011). Otra muy usual es incorporar un factor de ajuste a las líneas de control recurriendo a métodos clásicos de análisis matemático para la búsqueda de los valores óptimos de funciones (Sharma, 2003; Tang y Cheong, 2004; Zhang et al., 2006).

Sin embargo, independientemente de la alternativa aplicada, se observa en la literatura que el estudio de su 
desempeño se limita al análisis de LCP. Surgen dudas entonces sobre su desempeño real en relación con otras medidas de desempeño muy significativas. En particular, es difícil encontrar publicaciones, salvo en Sharma et al. (2003) aunque no en forma completa, que traten la carta $\mathrm{t}$ con $L C P$ no sesgada para monitorear tiempos TBE modelados según Weibull, muy útiles en contextos como mantenimiento y servicios.

\section{Objetivo y alcance del trabajo}

El objetivo del presente trabajo fue realizar un estudio comparativo del desempeño de las cartas bilaterales $t$ con y sin ajuste de sus límites de control con base en la metodología propuesta por Quintana et al. (2009) para monitorear la media de los tiempos TBE cuando se modelan mediante una distribución de probabilidad Weibull de dos parámetros. Esto fue motivado por la falta de publicaciones con análisis de su desempeño más allá de $L C P$, la repercusión de las cartas con límites de probabilidad en el ambiente Six Sigma, el alcance de los modelos Weibull en mantenimiento y el creciente interés por el uso de cartas de control en contextos más allá de producción. Mayores precisiones del comportamiento de estas cartas permiten entender las condiciones bajo las cuales son convenientes.

La distribución Weibull de dos parámetros depende de los parámetros de forma $\alpha$ y escala $\beta$. Dado que su media está en función de ambos, un cambio en ella puede generarse por un cambio en uno solo de sus parámetros o en ambos. Se contempla aquí el control de la media de TBE a través de $\beta$, ya que generalmente, un cambio en la operación del proceso provoca un cambio en $\beta$. A lo largo del trabajo, $\alpha$ se supone conocido. En cuanto a la carta con ajuste, el enfoque usado es la incorporación de un factor de ajuste a las líneas de control de la carta t.

La estructura del presente artículo es de la siguiente manera: en la primera sección (desarrollo) se presentan los supuestos en los que se basa el trabajo, las expresiones matemáticas de los límites de control y de las medidas $L C P$ y $L F A P$ para las cartas t con y sin ajuste con sus respectivas justificaciones, las medidas de resumen propuestas por Quintana et al. (2009) para evaluar el desempeño de una carta con una breve descripción del por qué de su elección y el cálculo de ellas para las cartas t con y sin ajuste. En la segunda sección (discusión y análisis de resultados) se presentan los gráficos que permiten comparar el desempeño de ambas cartas en términos de cada una de las medidas de resumen presentadas y el análisis de los resultados encontrados. En la tercera sección (conclusiones) se presentan los aportes del trabajo y futuras líneas de investigación.

\section{Desarrollo}

\section{Supuestos del trabajo}

Dado que el diseño de la carta t depende del modelo de probabilidad de las observaciones TBE y que tanto los parámetros del modelo Weibull como el corrimiento $D$ en la media pueden tomar cualquier valor positivo dentro de una escala continua, es necesario establecer algunos supuestos y restricciones:

- Los valores convenidos para $\alpha$ son: $1,2,3,4,6$ y 8

Este conjunto es suficiente para evaluar las diferencias en el comportamiento de las cartas y la mayoría de las aplicaciones. El caso $\alpha=1$ coincide con el modelo de probabilidad exponencial.

- Sea $\beta_{0}=1$ donde $\beta_{0}$ es el valor nominal de $\beta$ cuando $D=0$.

Este supuesto no causa pérdida de generalidad. Se justifica en que, si TBE se ajusta a un modelo Weibull con parámetros $\alpha$ y $\beta, T B E / \beta$ sigue un modelo Weibull con parámetros $\alpha$ y $\beta=1$.

- Sean $\mu_{0} y \sigma_{0}$ los valores nominales de la media y la desviación estándar de la variable TBE que se ajusta a un modelo de probabilidad Weibull con parámetros $\alpha$ y $\beta_{0}=$ 1:

$$
\begin{aligned}
\mu_{0} & =\Gamma\left(1+\frac{1}{\alpha}\right) \\
\sigma_{0} & =\sqrt{\Gamma\left(1+\frac{2}{\alpha}\right)-\Gamma^{2}\left(1+\frac{1}{\alpha}\right)}
\end{aligned}
$$

Es importante notar que estos valores dependen de $\alpha$.

- Sea y la media desplazada de TBE. Su forma de expresión usual es

$\mu=\mu_{0}+D$

donde $D=d \sigma_{0}$ con $d=0, \pm 0.25, \pm 0.50, \pm 1, \pm 1.5$ y \pm 2 . Cabe observar que $\mu$ depende tanto de $D$ como de $\alpha$.

- Los valores $\beta$ desplazados toman la forma

$$
\beta=1 \pm d \frac{\sigma_{0}}{\mu_{0}}
$$


Estos valores también dependen de $D$ y $\alpha$. La identidad (5) se justifica fácilmente: reemplazando $\mu$ por $\beta \Gamma\left(1+\frac{1}{\alpha}\right)$ en (4) y teniendo en cuenta (3) se despeja $\beta$. En particular y como es de esperar

Si $D=0$ entonces $\beta=\beta_{0}=1$ y $\mu=\mu_{0}$

Además, si $D>0$ entonces $\beta>1$ y $\mu>\mu_{0}$. Luego, en términos de TBE, el tiempo medio entre fallas es más amplio, lo cual implica que se produce un mejoramiento en el desempeño del equipo. En cambio, si $D<0, \beta<1$ y $\mu<\mu_{0}$; es decir, el tiempo medio entre fallas se ha reducido lo que equivale a un deterioro de su desempeño.

- Sea $p_{0}=0.0027$ siendo $p_{0}$ la probabilidad dada por (1)

Se convino en tomar este valor estándar para mantener la consonancia con las cartas de Shewhart.

Límites de control de la carta t sin ajuste

Las cartas con límites de probabilidad se caracterizan por la sencillez de su diseño. En el caso de la carta $t$, su estadístico de control es directamente TBE, la variable a monitorear. Ante cada falla del equipo o sistema se registra, mediante un punto en la gráfica, el tiempo transcurrido desde su última falla. Las líneas de control $L S$ y $L I$ se muestran en (10) y se justifican como sigue (Xie et al., 2002).

La expresión (1) se reescribe en función de $T B E$ como sigue

$p_{0}=P(T B E>L S$ si $D=0)+P(T B E<L I$ si $D=0)$

En cartas bilaterales, lo usual es considerar ambas probabilidades iguales. Chen (2011) considera probabilidades desiguales para que la carta tenga un desempeño con LCP insesgada. En este trabajo, para obtener una carta con esta propiedad se ha optado por la incorporación de un factor de ajuste a los límites de control manteniendo las probabilidades de (7) iguales

$P(T B E>L S$ si $D=0)=\frac{p_{0}}{2} \mathrm{P}(\mathrm{TBE}<\mathrm{LI}$ si $\mathrm{D}=0)=\frac{p_{0}}{2}$

Como TBE se ajusta a un modelo Weibull, su función de distribución de probabilidad acumulada es

$F(x)=P(T B E \leq x)=1-e^{-\left(\frac{x}{\beta}\right)^{\alpha}}$
A partir de (6) y (9), se resuelve (8). Al despejar $L S$ y $L I$ se obtiene

$L I=\left[\ln \left(\frac{2}{2-p_{0}}\right)\right]^{1 / \alpha} \quad L S=\left[\ln \left(\frac{2}{p_{0}}\right)\right]^{1 / \alpha}$

\section{LCP en la carta $t$ sin ajuste}

Como cada tiempo de falla se considera independiente de los anteriores, la variable $L C$ definida en (2) se ajusta a un modelo de probabilidad geométrico con parámetro $p$ definido como sigue para cada corrimiento $D$
$p=P($ la carta $\mathrm{t} \sin$ ajuste emita una señal de alarma verdadera)
$=P($ TBE $>L S)+P(T B E<L I)$

Considerando (9)

$p=1-e^{-\left(\frac{L l}{\beta}\right)^{\alpha}}+e^{-\left(\frac{L S}{\beta}\right)^{\alpha}}$

Se reemplaza $L S$ y $L I$ por (10)

$p=1-\exp \left(\frac{-\ln \left(\frac{2}{2-p_{0}}\right)}{\beta^{\alpha}}\right)+\exp \left(\frac{-\ln \left(\frac{2}{p_{0}}\right)}{\beta^{\alpha}}\right)$

De la naturaleza geométrica de la variable $L C$

$L C P=\frac{1}{p}$

Es importante notar que $L C P$ depende de $D$ y $\alpha$.

LFAP en la carta $t$ sin ajuste

Si $D=0$, considerando (6) se reemplaza $\beta$ por $\beta_{0}=1$ en (11) y se obtiene $p=p_{0}$. Al igual que $L C, L F A$ se ajusta a un modelo de probabilidad geométrico. Luego, reemplazando $p$ por $p_{0}$ en (12), sigue la primera igualdad de (13). Dado el supuesto $p_{0}=0.0027$, sigue la segunda igualdad de (13). Esto significa que, se espera en concepto de promedio, una falsa alarma cada 370 puntos graficados en la carta aún cuando $D=0$, análogo a lo que se espera en una carta estándar de Shewhart.

$L F A P=\frac{1}{p_{0}}=370.37$ 
Límites de control de la carta t con ajuste

$\mathrm{Al}$ igual que en la carta $\mathrm{t}$, el estadístico de control de la carta $t$ con ajuste es directamente TBE, la variable a monitorear. Para transformar la carta $t$ en una que tenga desempeño con $L C P$ no sesgada, la alternativa elegida aquí es multiplicar los límites (10) por una constante apropiada, el factor $f$ de ajuste:

$$
L l=f \cdot\left[\ln \left(\frac{2}{2-q_{0}}\right)\right]^{1 / \alpha} \quad L S=f \cdot\left[\ln \left(\frac{2}{q_{0}}\right)\right]^{1 / \alpha}
$$

donde $q_{0}$ es la probabilidad admisible y fijada de antemano de que la carta $t$ con ajuste emita una señal de falsa alarma. El rol del factor $f$ es provocar que la curva $L C P$ alcance su máximo en $D=0$, es decir en $\beta_{0}=1$ (Sharma et al., 2003). Esto implica

$$
\left.\frac{d L C P}{d \beta}\right|_{\beta=\beta_{0}}=0
$$

La búsqueda del factor $f$ requiere de la resolución de (15). Para ello, es necesario que $L C P$ se exprese en función de $\beta$. La variable $L C$ de la carta $t$ con ajuste sigue también un modelo de probabilidad geométrico con parámetro $q$ definido para cada corrimiento $D$ por:

$q=P($ la carta $\mathrm{t}$ con ajuste emita una señal de alarma $)$

$$
=P(T B E>L S)+P(T B E<L I)
$$

Considerando (9)

$$
q=1-e^{-\left(\frac{L l}{\beta}\right)^{\alpha}}+e^{-\left(\frac{L S}{\beta}\right)^{\alpha}}
$$

Se reemplaza $L S$ y $L I$ por (14) y se obtiene

$$
q=1-\exp \left(\frac{-f^{\alpha} \ln \left(\frac{2}{2-q_{0}}\right)}{\beta^{\alpha}}\right)+\exp \left(\frac{-f^{\alpha} \ln \left(\frac{2}{q_{0}}\right)}{\beta^{\alpha}}\right)
$$

De la naturaleza geométrica de la variable $L C$

$$
L C P=\frac{1}{q}
$$

Tomando en cuenta las dos últimas expresiones se resuelve (15). Al despejar $f$ se obtiene (17). Aplicando cri- terios clásicos de análisis matemático como el de la derivada primera, se comprueba que $L C P$ presenta su máximo en $\beta_{0}=1$.

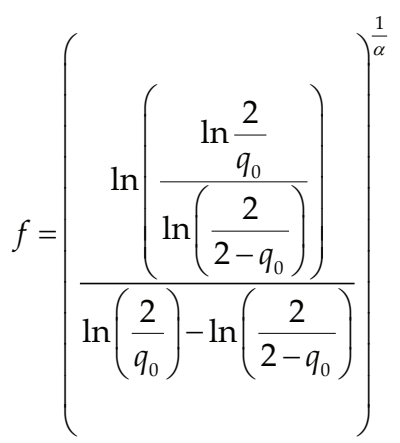

LCP en la carta t con ajuste

Como se explicó anteriormente, de la naturaleza geométrica de la variable $L C$

$L C P=\frac{1}{q}$

donde $q$ está dada por (16). Al igual que con la carta $\mathrm{t}$ sin ajuste, $L C P$ depende de $D$ y $\alpha$.

\section{LFAP en la carta t con ajuste}

Si $D=0$, teniendo en cuenta (6) se reemplaza $\beta$ por $\beta_{0}=$ 1 y $f$ por (17) en (16). Se obtiene $q=q^{*}$, la probabilidad real de que la carta t con ajuste emita una señal de falsa alarma dada por:

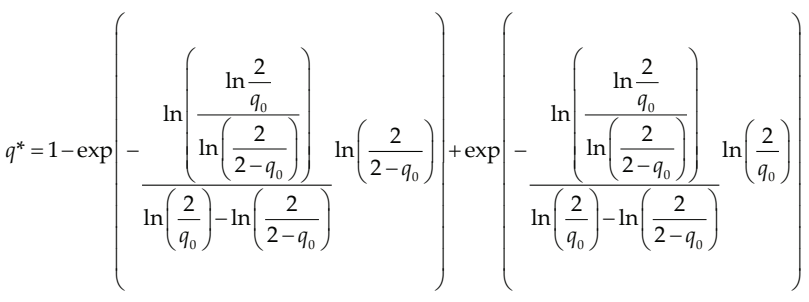

De la naturaleza geométrica de $L F A$ y reemplazando $q$ por (19) en (18) se obtiene

$L F A P=\frac{1}{q^{*}}$

Desafortunadamente, la incorporación del factor de ajuste causa un inconveniente. A diferencia de lo que 
ocurre con la carta $t$ sin ajuste y contrario a lo que es de esperar, de (19) se observa que $q^{*} \neq q_{0}$. Esto significa que, aún fijando inicialmente una probabilidad $q_{0}$ de falsa alarma en 0.0027 como en la carta $t$ sin ajuste, la probabilidad real de que la carta $t$ con ajuste emita una falsa alarma $\left(q^{*}\right)$ difiere de 0.0027. Luego, de (20) resulta que $L F A P \neq 370.37$, lo que provoca que las cartas $\mathrm{t}$ con $\mathrm{y}$ sin ajuste no sean comparables cuando se fijan $p_{0}$ y $q_{0}$ en el mismo valor dado que las LFAP de ambas cartas son desiguales.

Afortunadamente, de (19) se observa que $q^{*}$ es una función que depende de $q_{0}$, pero no de $\alpha$. Esta ventaja permite remendar el problema modificando estratégicamente el valor de $q_{0}$. La corrección consiste en fijar $q_{0}$ en un valor conveniente tal que $q^{*}=0.0027$. La engorrosa expresión de $q^{*}$ en (19) requiere que se trabaje numéricamente con la ayuda de algún software adecuado como EXCEL o MATHEMATICA:

Para alcanzar $q^{*}=0.0027$ es necesario tomar

$$
q_{0}=0.003721213378
$$

Luego

$$
\begin{aligned}
\text { LFAP } & =\frac{1}{q^{*}}=370.37 \text { siempre y cuando se tome } \\
q_{0} & =0.003721213378
\end{aligned}
$$

\section{Desempeño de las cartas}

El desempeño de las cartas se evaluó siguiendo la metodología propuesta por Quintana et al. (2009) que contempla el uso de una terna de medidas de resumen integrada por un promedio, una medida de variabilidad lateral y una proporción, diferente para cada estado posible $(D=0$ y $D \neq 0)$, a partir de los argumentos que se describen brevemente a continuación.

La alta variabilidad de $L C$ y $L F A$ hacen que, el uso de $L C P$ y $L F A P$ como únicas medidas del desempeño de una carta, sean insuficientes. El coeficiente de variación de una variable aleatoria, métrica usual en estadística para medir su variabilidad relativa, es igual al cociente entre la desviación estándar y la media de la variable. Sin embargo, el coeficiente de variación (VT) de LCo $L F A$ no es una medida completamente adecuada para evaluar la dispersión en la velocidad de respuesta de una carta, dado que LC y LFA presentan una acentuada asimetría. Para solucionar este problema, Quintana et al. (2009) demuestran que el coeficiente VT puede ser descompuesto en dos componentes discriminando las
$L F A$ inferiores a $L F A P$ de las superiores si $D=0$. Asimismo, si $D \neq 0$, discrimina las $L C$ inferiores a $L C P$ de las superiores:

$V T^{2}=P C C^{*} V I^{2}+P C L^{*} V D^{2}$

donde

$P C C=$ proporción de corridas más cortas (más tempra nas) que $L C P$ (o LFAP)

$P C L=$ proporción de corridas más largas (más tardías) que LCP (o LFAP)

$\mathrm{PCC}+\mathrm{PCL}=1$

$V I$ = variabilidad relativa o coeficiente de variación a la izquierda de $L C P$ (o LFAP)

$V D=$ variabilidad relativa o coeficiente de variación a la derecha de $L C P$ (o LFAP)

Importancia de las variabilidades laterales según el estado del proceso

Debido a la naturaleza asimétrica de las variables $L F A$ y $L C$, las variabilidades laterales no contribuyen en iguales proporciones a $V T$ y no revisten la misma importancia para ambos estados $(D=0$ y $D \neq 0)$ si se tiene en cuenta lo que mide una y otra variable. No toda la variabilidad contenida en $V T$ es perjudicial. Teniendo en cuenta que, la carta es más eficiente cuando, en lo que respecta a $L C P$ y $L F A P$, mayor es $L F A P$ si $D=0$ y menor es $L C P$ si $D \neq 0$, se deduce:

- Si $D=0$, las corridas con longitudes superiores a LFAP contribuyen a engrosar VT favoreciéndola. El hecho de que $V D$ sea grande no es motivo de preocupación. VI es el único componente realmente perjudicial que incrementa $V T$, dado que proviene de las corridas con longitudes inferiores a LFAP.

- Si $D \neq 0$, la situación es a la inversa. Las corridas con longitudes inferiores a LCP son bienvenidas. Luego, el hecho de que VI sea grande no es un problema. VD es el único componente realmente preocupante que incrementa $V T$, dado que proviene de las corridas con longitudes superiores a $L C P$.

Medidas de resumen para evaluar el desempeño de una carta de control

De lo analizado se concluye que, cuando $D=0, L F A P$ y $V I$ son indicadores adecuados para evaluar el desempeño de la carta. En cambio, $L C P$ y $V D$ son aptos para $D \neq 0$. Resulta conveniente completar la primera dupla 
anterior con PCC para evaluar el grado de incidencia de valores altos de VI. Análogamente, para $D \neq 0$, la dupla se completa con PCL para evaluar el grado de incidencia de valores altos de $V D$. En resumen, para evaluar el desempeño de una carta, se proponen las ternas:

- $L F A P, V I$ y PCC para $D=0$

- $L C P, V D$ y $P C L$ para cada corrimiento $D \neq 0$

La carta tendrá mejor desempeño cuanto mayor sea su LFAP y menor sea el resto de las medidas para un rango de valores $D$ de interés. Análogo a las cartas de Shewhart, cada tiempo de falla se considera independiente de los anteriores. Luego, tanto LFA como LC siguen el modelo de probabilidad geométrico. En símbolos:

$P(X=x)=(1-\pi)^{x-1} \cdot \pi$

donde

$X=L F A$ o $L C$ según sea $D=0$ o $D \neq 0$, respectivamente

$\pi=p_{0} \mathrm{o} q^{*}$ para la carta $\mathrm{t} \sin$ ajuste o con ajuste, respectivamente si $X=L F A$

$\pi=p$ o $q$ para la carta $t$ sin ajuste o con ajuste, respecti vamente si $X=L C$

Luego, su función de distribución acumulada es una suma finita de una progresión geométrica:

$F(x)=P(X \leq x)=1-(1-\pi)^{[x]}$

donde $[x]$, parte entera de $x$.

Las expresiones de las medidas de resumen propuestas surgen a partir de la demostración de la igualdad (22) en el trabajo de Quintana et al. (2009) (el operador $P(\cdot)$ significa probabilidad):

- $\operatorname{Si} D=0$ :

$P C C_{0}=P(L F A<L F A P)$

$V I_{0}=\frac{\sqrt{\sum_{x<L F A P}(x-L F A P)^{2} \cdot \frac{P(L F A=x)}{P C C_{0}}}}{L F A P}$

El numerador de (25) es la desviación estándar a la izquierda de $L F A P$.

- Si $D \neq 0$ :

$P C L=P(L C>L C P)$
$V D=\frac{\sqrt{\sum_{x>L C P}(x-L C P)^{2} \cdot \frac{P(L C=x)}{P C L}}}{L C P}$

El numerador de (27) es la desviación estándar a derecha de $L C P$.

$P C C_{0}$ para $D=0$

La primera igualdad de (28) se justifica a partir de (24) teniendo en cuenta (23). De (13) y (21), LFAP = 370.3 para ambas cartas. De lo visto, $p_{0}=q^{*}=0.0027$ (siempre y cuando se fije $q_{0}$ en 0.003721213378$)$. Luego, sigue la segunda igualdad de (28).

$P C C_{0}=1-(1-\pi)^{[L F A P-1]}=0.6312$

donde $\pi=p_{0}$ o $q^{*}$ según se trate de la carta $t$ sin o con ajuste, respectivamente.

$V I_{0}$ para $D=0$

De (13), (21) y (28), $L F A P=370.37$ y $P C C_{0}=0.6312$ para ambas cartas. Luego de trabajar numéricamente (25) con el software MATHEMATICA, se obtiene:

$V I_{0}=0.6461$

$\mathrm{PCL}$ para $\mathrm{D} \neq 0$

A partir de (23), (26) se convierte en

$P C L=(1-\pi)^{[L C P]}$

donde $\pi=p$ o $q$ según se trate de la carta $t$ sin o con ajuste, respectivamente. Es importante notar que PCL depende de $D$ y $\alpha$.

VD para $\mathrm{D} \neq 0$

Si bien (27) define a $V D$, involucra el engorroso cálculo de una suma infinita. Una alternativa más sencilla es despejar VD de (22)

$V D=\sqrt{\frac{V T^{2}-(1-\mathrm{PCL}) \cdot V I^{2}}{P C L}}$

Dado que $L C$ es una variable geométrica con parámetro $\pi$, su media es $1 / \pi$ y su desviación estándar, $\sqrt{1-\pi} / \pi$. Luego, el coeficiente de variación total VT es 
$V T=\sqrt{1-\pi}$ con $\pi=p$ o $q$ para la carta sin ajuste o con ajuste, respectivamente

La expresión de VI surge a partir de la demostración de la igualdad (22) en el trabajo de Quintana et al. (2009):

$$
V I=\frac{\sqrt{\sum_{x \leq L C P}(x-L C P)^{2} \cdot \frac{P(L C=x)}{1-P C L}}}{L C P}
$$

Al igual que $P C L$, cabe destacar que el valor de $V D$ depende de $D$ y $\alpha$.

\section{Gráficos}

Es importante notar que los cálculos de $L C P, P C L$ y $V D$ para ambas cartas dados por (12), (18), (29) y (30) se deben realizar para cada par de valores $(D, \alpha)$ con $D \neq 0$, contemplados en la sección supuestos del trabajo. Dada la numerosa cantidad de combinaciones posibles y para facilitar el análisis de las conclusiones, se presentan gráficos apropiados. En las figuras 1, 4 y 5 se aprecian los resultados de la carta $t$ sin ajuste. En la figura 2, los de $L C P$ para la carta $t$ con ajuste. Para comparar las cartas, se grafican las relaciones:

$L C P$ con ajuste / LCP sin ajuste (figura 3) $V D$ con ajuste / $V D$ sin ajuste (figura 6) y $P C L$ con ajuste / PCL sin ajuste (figura 7)

\section{Discusión y análisis de resultados}

Para $D \neq 0$, el ajuste de los límites de control ha dado sus frutos como se observa en las figuras 1 y 2 . En la carta $t$ sin ajuste (figura 1 ), para pequeños deterioros del equipo o sistema ( $D<0$ muy pequeño), $L C P$ supera significativamente al valor $L F A P$ preasignado de 370 puntos. Esto significa que, ante pequeños deterioros, la carta tarda más en emitir una señal que cuando no se producen. De la figura 2, la ventaja del ajuste es evidente, mantiene a $L C P$ por debajo de ese nivel, cualesquiera sean $D$ y $\alpha$.

La naturaleza asimétrica de Weibull hace que, para valores $D$ de igual magnitud pero distinto sentido, las $L C P$ de ambas cartas difieran significativamente, aunque con menor intensidad en la carta $t$ con ajuste y ser mayores para $D<0$. Esto significa que, a igual tenor de $D$, ambas cartas son mucho más veloces para detectar mejoramientos que deterioros. No obstante, estas discrepancias se suavizan conforme aumenta la magnitud de $D$. Se observa además que, para la mayoría de los corrimientos $D$, el valor $L C P$ de cada carta no difiere significativamente de un valor $\alpha$ a otro, para los contemplados en este trabajo.

\section{LCP carta Weibull sin ajuste}

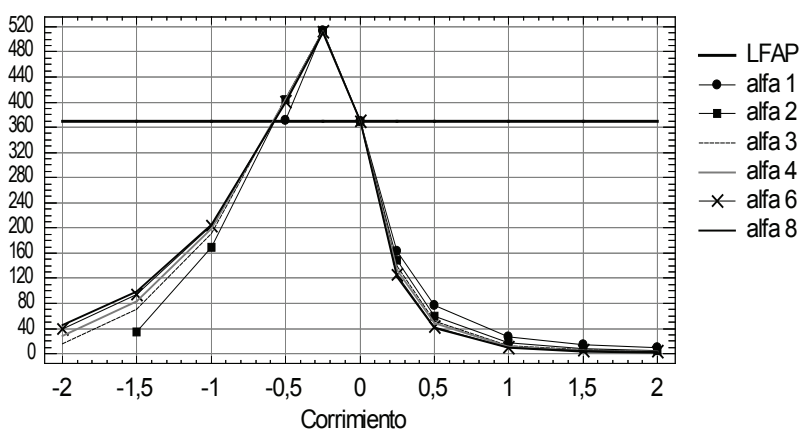

Figura 1. LCP cuando $D \neq 0$ para la carta $t$ sin ajuste

\section{LCP carta Weibull con ajuste}

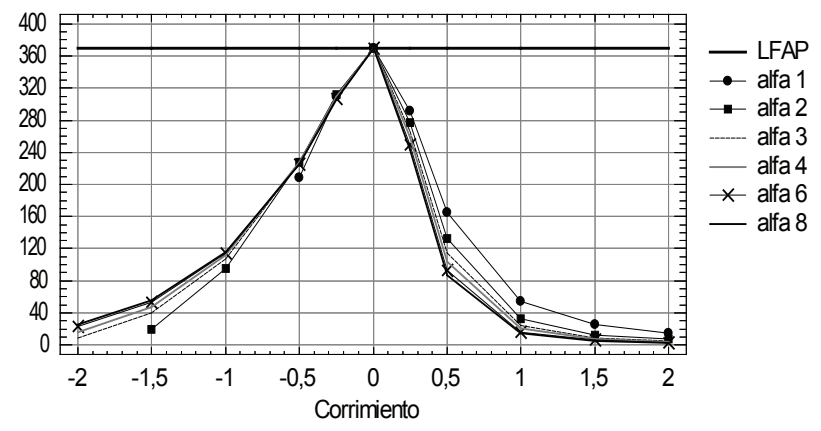

Figura 2. LCP cuando $\mathrm{D} \neq 0$ para la carta t con ajuste

El comportamiento de $L C P$ en la carta t con ajuste varía según el signo de $D$ (figura 3). Para $D<0$ (deterioros), es claramente ventajosa, significativamente más sensible, más rápida que la carta $t \sin$ ajuste. Su $L C P$ es aproximadamente $40 \%$ inferior a la del caso sin ajuste.

En cambio, para $D>0$ (mejoramientos), la carta t con ajuste se torna perjudicial. Su $L C P$ es significativamente superior a la de la carta $t$ sin ajuste, especialmente para corrimientos $D>0$ muy pequeños, aunque, la magnitud del incremento disminuye conforme aumenta $D$ y también con $\alpha$. En conclusión, la carta t con ajuste trae como contrapartida una carta menos sensible, más lenta que la carta $\mathrm{t}$ sin ajuste para detectar mejoramientos del equipo o sistema.

En cuanto a las otras medidas de desempeño, en la carta $t \sin$ ajuste se aprecia una muy elevada variabilidad derecha (figura 4). Para deterioros $(D<0)$, es del orden de $140 \%$ aproximadamente. Para mejoramientos $(D>0)$, disminuye levemente hasta alcanzar un mínimo 
de $110 \%$. Esos altos niveles de VD están acompañados por una proporción de corridas largas PCL de 37\% aproximadamente, para $D<0$, y oscilando entre $30 \%$ y $40 \%$, para $D>0$ (figura 5).

\section{Relación: LCP con ajuste / LCP sin ajuste}

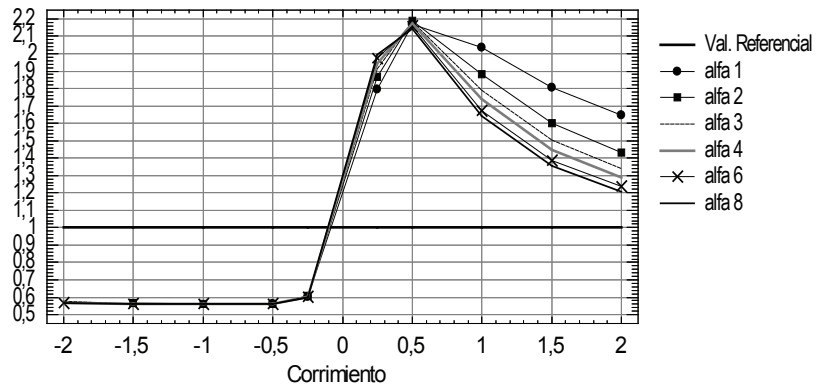

Figura 3. Relación $\frac{L C P \text { con ajuste }}{L C P \text { sin ajuste }}$ para $D \neq 0$

\section{VDer carta Weibull sin ajuste}

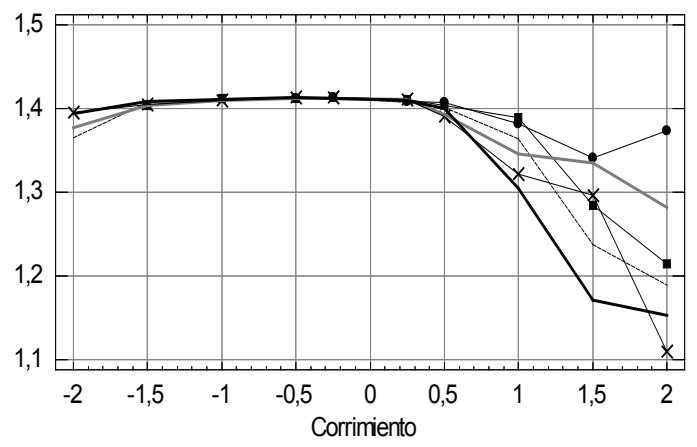

Figura 4. VD cuando $D \neq 0$ para la carta $t$ sin ajuste

\section{PCL carta Weibull sin ajuste}

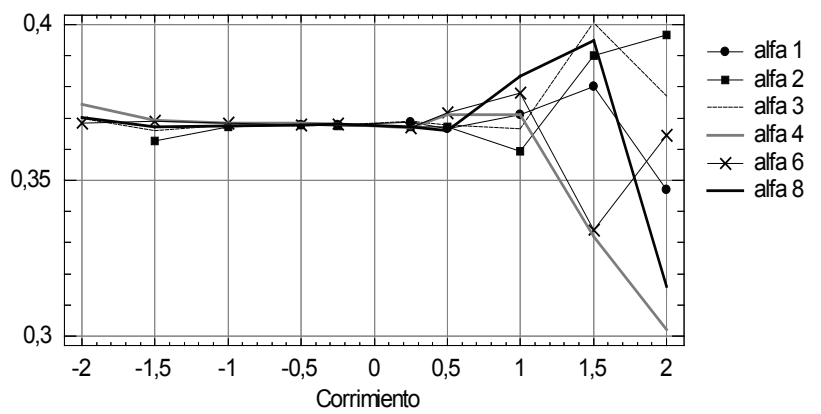

Figura 5. $\mathrm{PCL}$ cuando $\mathrm{D} \neq 0$ para la carta $t$ sin ajuste

La carta $\mathrm{t}$ con ajuste, para $D<0$, mantiene los mismos niveles elevados de $V D$ de la carta $t$ sin ajuste y también casi iguales valores PCL. Similar comportamiento se aprecia para corrimientos positivos de magnitud pequeña $(0<D<1 \sigma)$ (figuras 6 y 7$)$.
Para la mayoría de los corrimientos positivos de mayor magnitud $(D \geq 1 \sigma)$, el incremento en $L C P$ de la carta con ajuste está acompañado de un leve incremento en los valores $V D$ (inferior a 20\%) (figura 6). Sin embargo, este aumento no es preocupante dado que está atenuado por una reducción en PCL (inferior a 20\%) (figura 7). Es decir, si bien la $L C$ carta $t$ con ajuste es más variable que la del caso sin ajuste, menor es la probabilidad de que presente una corrida más larga que el promedio.

En resumen, el ajuste de los límites de control solo alcanza para corregir aquellos valores $L C P$ de la carta $t$ sin ajuste que están por encima del valor $L F A P$ preasignado, pero no para disminuir sus elevados niveles de $V D$.

\section{Relación VDer con ajuste/VDer sin ajuste}

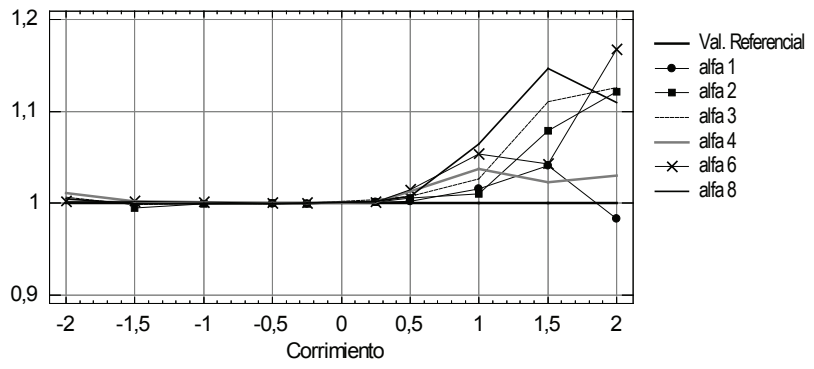

Figura 6. Relación $\frac{V D \text { con ajuste }}{V D \text { sin ajuste }}$ para $D \neq 0$

\section{Relación: PCL con ajuste / PCL sin ajuste}

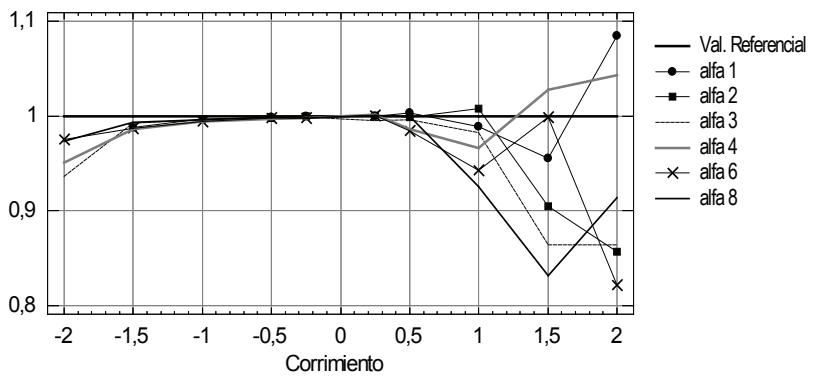

Figura 7. Relación $\frac{P C L \text { con ajuste }}{P C L \text { sin ajuste }}$ para $D \neq 0$

\section{Conclusiones}

El principal objetivo de este trabajo fue realizar un análisis y comparación de las ventajas y desventajas del uso de cartas de relativa sencillez que surgieron en años recientes en concordancia con la evolución de la metodología Six Sigma y de particular interés en mantenimiento, evaluando no solo la tradicional $L C P$, sino también otras facetas poco exploradas de la velocidad de respuesta de una carta de control. 
Atendiendo a la velocidad media de respuesta, si el objetivo principal del monitoreo del equipo o sistema recae en detectar deterioros, se recomienda la carta $t$ con ajuste. En cambio, cuando la prioridad es detectar mejoramientos, la carta $t$ sin ajuste es la mejor opción. Sin embargo, en un programa Six Sigma, el interés recae en detectar tanto deterioros como mejoramientos del proceso, para no desatender información valiosa que pueda dar lugar a una oportunidad de mejora. De usar estas cartas, se sugiere su uso simultáneo.

No obstante, la velocidad de respuesta de ambas cartas es muy variable de corrida en corrida. Con frecuencia el costo que se paga por una carta con diseño de relativa sencillez, como las presentadas en este trabajo, es la obtención de una carta menos veloz y más variable que otras disponibles en la literatura, entre las que se destaca, la carta CUSUM Weibull (Quintana y García, 2010). Es bien conocido que las cartas CUSUM son rápidas en la detección de corrimientos muy pequeños aunque su diseño es más complejo.

Desde el punto de vista operacional, las cartas t con y sin ajuste son más recomendables que la carta CUSUM Weibull. La primera razón es que el diseño de las cartas $\mathrm{t}$ requiere del proceso menos información que CUSUM. Esto es particularmente útil en situaciones donde no se tiene plena claridad de cuál magnitud del corrimiento $D$ es la más crítica; es decir, a partir de qué valor $D$ se pueden obtener serios perjuicios en el proceso si el cambio no es detectado a tiempo (requerimiento indispensable para diseñar una carta CUSUM). Esto ocurre, por ejemplo, en la puesta en marcha de un equipo o sistema nuevo o cuando no se cuentan con suficientes registros de su historial de funcionamiento. Como consecuencia, para determinar los límites de control, es más simple llevarlo a cabo con la carta t que con CUSUM. La segunda razón es que el cómputo de las medidas de desempeño de la carta es más sencillo en las cartas $t$, dado que se disponen de fórmulas precisas para ello. Por el contrario, para la carta CUSUM, es necesario recurrir a algoritmos aproximados.

Sin pretender hacer una enumeración exhaustiva, otras alternativas existentes en la literatura son: la carta $\mathrm{t}_{\mathrm{r}}$ (extensión de la carta $\mathrm{t}$ ), las cartas no paramétricas y las que se basan en el método de la varianza ponderada. Se dispone de estudios de sus desempeños aunque generalmente solo en términos de LCP y ATS. En el caso de las cartas no paramétricas, pocas publicaciones hacen comparaciones con cartas diseñadas para procesos Weibull y menos si se trata de aplicaciones a mantenimiento. En cambio, en el caso de las cartas basadas en el método de la varianza ponderada, la literatura evidencia interés sobre su aplicación a procesos Weibull, aun- que con análisis limitados de su desempeño. Por ello, el análisis de las características, bondades y limitaciones de las cartas alternativas nombradas, más allá de las medidas convencionales de desempeño, es de sumo interés para los autores de este trabajo en una futura investigación, con el objeto de comparar con las cartas t presentadas aquí y orientar sobre la alternativa más conveniente a las condiciones y limitaciones de un proceso, con especial énfasis en mantenimiento.

\section{Referencias}

Alemi F. Tukey's Control Chart. Quality Management In Health Care, volumen 13 (número 4), octubre-diciembre 2004 [en linea] [fecha de consulta: 12 de marzo de 2014]. Disponible en: http://www.openonlinecourses.com/cqi/Tukey\%20Control\%20Chart\%20Alemi.pdf

Bakir S.T. A Distribution-Free Shewhart Quality Control Chart Based on Signed-Ranks. Quality Engineering, volumen 16 (número 4), 2004 [en linea] [fecha de consulta: 14 de marzo de 2014]. Disponible en: http://www.tandfonline.com/doi/abs/ 10.1081/QEN-120038022

Castagliola P., Giovanni C., Fichera S. Handbook of Engineering Statistics, Londres, Springer-Verlag, 2006 [en linea] [fecha de consulta: 23 de septiembre de 2013], capítulo 17, Monitoring Process Variability Using EWMA. Disponible en: http://link.springer.com/referenceworkentry/10.1007/978-1-84628-288-1_17

Castagliola P., Khoo M. A Synthetic Scaled Weighted Variance Control Chart for Monitoring the Process Mean of Skewed Populations. Communications in Statistics-Simulation and Computation, volumen 38, 2009 [en linea] [fecha de consulta: 12 de marzo de 2014]. Disponible en: http://peer.ccsd.cnrs.fr/ d ocs / $00 / 51 / 43$ / 50 / P D F / P E E R_st a ge 2_ 10 . 1080\%252F03610910903072409.pdf

Chakraborti S. Run Length Distribution and Percentiles: The Shewhart Chart with Unknown Parameters. Quality Engineering, volumen 19 (número 2), marzo 2007 [en linea] [fecha de consulta: 23 de septiembre de 2013]. Disponible en: http://www.tandfonline.com/doi/abs/10.1080/ 08982110701276653

Chakraborti S., Van de Wiel M. Institute of Mathematical Statistics Collections: Beyond Parametrics in Interdisciplinary Research: Festschrift in Honor of Professor Pranab K. Sen, volumen 1, USA, Balakrishnan N., Peña E.A., Silvapulle M.J., 2008 [en linea] [fecha de consulta: 14 de marzo de 2014], pp. 156172, A Nonparametric Control Chart Based on the MannWhitney Statistic. Disponible en: http://projecteuclid.org/ euclid.imsc/1207058271

Chen P.W., Cheng C.S. On Statistical Design of the Cumulative Quantity Control Chart for Monitoring High Yield Processes. Communications in Statistics- Theory and Methods, volumen 40 (número 11), marzo 2011 [en linea] [fecha de consulta: 23 de 
septiembre de 2013]. Disponible en: http://www.tandfonline. com/doi/abs/10.1080/03610920903391329

Govindaraju K., Lai C.D. Run Length Variability and Three Sigma Control Limits. Economic Quality Control, volumen 19 (número 2), octubre 2004 [en linea] [fecha de consulta: 23 de septiembre de 2013]. Disponible en: http://www.degruyter.com/ view/j/ eqc.2004.19.issue-2/eqc.2004.175/eqc.2004.175.xml

Govindaraju K. Statistical Performance of Control Charts. Economic Quality Control, volumen 20 (número 1), marzo 2005 [en linea] [fecha de consulta: 23 de septiembre de 2013]. Disponible en: http://www.degruyter.com/view/j/ eqc.2005.20.issue-1/eqc.2005.5/eqc.2005.5.xml

Gutiérrez-Pulido H., De la Vara Salazar R. Control estadístico de calidad y seis sigma, 2aㅡ ed., México, Mc Graw Hill Interamericana Editores, 2009, pp. 4-6

Hawkins D., Olwell D. Cumulative Sum Charts and Charting for Quality Improvement, New York, Springer-Verlag, 1998, pp. 83-104

Khoo M.B.C., Atta A.M.A., Chen C-H. Proposed and S Control Charts for Skewed Distributions, en: International Conference on Industrial Engineering and Engineering Management. Proceedings IEEM IEEE, Hong Kong, China, 2009 [en linea] [fecha de consulta: 10 de marzo de 2014] Disponible en: http://ieeexplore.ieee.org/xpls/abs_all.jsp?arnumber=5373327\&tag=1

Lai C.D., Chan L.Y., Xie M. Distribution Of Runs In A Two-Stage Process Monitoring Model. Communications in Statistics-Simulation and Computation, volumen 30 (número 3), 2001 [en linea] [fecha de consulta: 23 de septiembre de 2013]. Disponible en: http://www.tandfonline.com/doi/abs/10.1081/SAC-100105078

Lai C.D., Govindaraju K. Reduction of Control-Chart Signal Variablity for High-Quality Processes. Journal of Applied Statistics, volumen 35 (número 6), mayo 2008 [en linea] [fecha de consulta: 23 de septiembre de 2013]. Disponible en: http:// www.tandfonline.com/ doi/abs/10.1080/02664760801921232

Lai C.D., Pra-Murphy D.N., Xie M. Handbook of Engineering Statistics, London, Springer-Verlag, 2006 [en linea] [fecha de consulta: 23 de septiembre de 2013], capítulo 3, Weibull Distributions and Their Applications. Disponible en: http://link.springer. com/referenceworkentry/10.1007/978-1-84628-288-1_3

Li Z., Zhou S., Sievenpiper C., Choubey S. Statistical Monitoring of Time-to-Failure Data Using Rank Tests. Quality and Reliability Engineering International, volumen 28, 2012 [en linea] [fecha de consulta: 11 de marzo de 2014]. Disponible en: http:// homepages.cae.wisc.edu/ zhous/papers/qre1248.pdf

Linderman K., McKone-Sweet K.E., Anderson J.C. An Integrated Systems Approach to Process Control and Maintenance. European Journal of Operational Research, volumen 164 (número 2), 2005 [en linea] [fecha de consulta: 10 de marzo de 2014]. Disponible en: http://www.sciencedirect.com/science/article/pii/ S0377221704000219\#

Liu J.Y., Xie M., Goh, T.N., Sharma P.R. A Comparative Study of Exponential Time between Events Charts. Quality Technology and
Quantitative Management, volumen 3 (número 3), 2006 [en linea] [fecha de consulta: 23 de septiembre de 2013]. Disponible en: http://web.it.nctu.edu.tw/ qtqm/qtqmpapers/ 2006V3N3/2006V3N3_F8.pdf

Luceño A., Puig-Pey J. Evaluation of the Run-Length Probability Distribution for Cusum Charts: Assessing Chart Performance. Technometrics, volumen 42 (número 4), 2000 [en linea] [fecha de consulta: 23 de septiembre de 2013]. Disponible en: http://amstat.tandfonline.com/doi/abs/10.1080/00401706.200 0.10485714

Quintana A., García L., Casal, R. Medidas de resumen para evaluar la performance de una carta de control para cada estado de un proceso. Su aplicación a cartas de Shewhart y Cusum. Revista de la Escuela de Perfeccionamiento en Investigación Operativa, Argentina, volumen XVII (número 30), mayo 2009: 39-58.

Quintana A., García L. Diseño y performance de la carta de control Cusum Weibull bilateral para individuales. Comparación con la carta Cusum normal ante la presencia de procesos Weibull, en: Encuentro Regional Argentino Brasileño de Investigación Operativa (II, 2010, Tandil, Argentina). Anales XXIII ENDIOXXI EPIO-II ERABIO, Tandil, Argentina, Universidad Nacional del Centro de la Provincia de Buenos Aires, Escuela de Perfeccionamiento en Investigación Operativa (EPIO), Sociedade Brasileira de Pesquisa Operacional (SOBRAPO), 2010, 213 a 232.

Quintana A., García L., Casal R. Variabilidad lateral para evaluar la velocidad de respuesta de una carta de control de procesos. Revista Ingeniería Industrial, Universidad del Bío-Bío, Chile, volumen 10 (número 2), 2011 [en linea] [fecha de consulta: 23 de septiembre de 2013]. Disponible en: http://www.ici.ubiobio.cl/revista/index. php?option=com_content\&task=view\&i $\mathrm{d}=35 \&$ Itemid $=36$

Radson D., Boyd A.H. Graphical Representation of Run Length Distributions. Quality Engineering, volumen 17 (número 2), 2005 [en linea] [fecha de consulta: 23 de septiembre de 2013]. Disponible en: http://www.tandfonline.com/doi/abs/ 10.1081/ QEN-200056484

Ryan T. Statistical Methods for Quality Improvement, 3a ed., Nueva Jersey, John Wiley \& Sons, 2011 [en linea] [fecha de consulta: 23 de septiembre de 2013], capítulo 4, Control Charts for Measurements With Subgrouping (for One Variable). Disponible en: http://onlinelibrary.wiley.com/book/10.1002/ 9781118058114

Santiago E., Smith J. Control Charts Based on the Exponential Distribution: Adapting Runs Rules for the t chart. Quality Engineering, volumen 25 (número 2), febrero 2013 [en linea] [fecha de consulta: 23 de septiembre de 2013]. Disponible en: http:// www.tandfonline.com/doi/abs/10.1080/08982112.2012.740646

Sharma P.R. A Study of Properties and Applications of Control Charts for High Yield Processes, tesis (doctor en filolosofía), Singapur, The National University of Singapore, Department of Industrial and Systems Engineering, 2003, 240 pp. [en linea] [fecha de consulta: 23 de septiembre de 2013] Disponible en: http:// 
scholarbank.nus.edu.sg/bitstream/ handle/10635/14197/SharmaPR.pdf?sequence=1

Sharma P.R., Xie M., Goh T.N. Reliability Modeling, Analysis and Optimization. Series on Quality, Reliability and Engineering Statistics, volumen 9, Singapur, World Scientific Publishing Co., 2006 [en linea] [fecha de consulta: 23 de septiembre de 2013], capítulo 3, Monitoring Inter-Arrival Times with Statistical Control Charts. Disponible en: http://www.worldscientific. com/doi/abs/10.1142/9789812707147_0003

Surucu B., Sazak H. Monitoring Reliability for a Three Parameter Weibull Distribution. Reliability Engineering and System Safety, volumen 94 (número 2), febrero 2009 [en linea] [fecha de consulta: 23 de septiembre de 2013]. Disponible en: http://www. sciencedirect.com/science/article/pii/S0951832008001737

Tang L.C., Cheong W.T.. Cumulative Conformance Count Chart with Sequentially Updated Parameters. IIE Transactions, volumen 36 (número 9), 2004 [en linea] [fecha de consulta: 23 de septiembre de 2013]. Disponible en: http://www.tandfonline. com/doi/ abs/10.1080/07408170490473024

Xie M., Goh T.N., Ranjan P. Some Effective Control Chart Procedures for Reliability Monitoring. Reliability Engineering and System Safety, volumen 77 (número 2), agosto 2002 [en linea] [fecha de consulta: 23 de septiembre de 2013]. Disponible en: http:// www.sciencedirect.com/science/article/pii/S0951832002000418

Xie M., Goh T. Handbook of Engineering Statistics, Londres, SpringerVerlag, 2006 [en linea] [fecha de consulta: 23 de septiembre de 2013], capítulo 16, Some Statistical Models for the Monitoring of High-Quality Processes. Disponible en: http://link.springer. com/referenceworkentry/10.1007/978-1-84628-288-1_16
Zhang C.W., Xie M., Goh T.N. Design of Exponential Control Charts Using a Sequential Sampling Scheme. IIE Transactions, volumen 38 (número 12), noviembre 2006 [en linea] [fecha de consulta: 23 de septiembre de 2013]. Disponible en: http:// www.tandfonline.com/doi/abs/10.1080/07408170600728905

Zhao Y., Liang G., Zhou X. Equipment Spare Parts Failure Preventive Control Based on Control Charts, en: International Conference on Management Science \& Engineering $\left(19^{\text {th }}, 2012\right.$, Dallas, USA). Proceedings ICMSE, Dallas, USA, 2012, 596 a 600 [en linea] [fecha de consulta: 10 de marzo de 2014] Disponible en: http://ieeexplore.ieee.org/xpl/articleDetails. jsp?arnumber $=6414241$

\section{Este artículo se cita: \\ Citación estilo Chicago \\ Quintana, Alicia Esther, María Virginia Pisani, Ricardo Néstor Ca- sal. Desempeño de cartas de control estadístico con límites bilate- rales de probabilidad para monitorear procesos Weibull en mantenimiento. Ingeniería Investigación y Tecnología, XVI, 01 (2015): 143-156.}

\section{Citación estilo ISO 690}

Quintana- A.E., Pisani- M.V., Casal- R.N. Desempeño de cartas de control estadístico con límites bilaterales de probabilidad para monitorear procesos Weibull en mantenimiento. Ingeniería Investigación y Tecnología, volumen XVI (número 1), enero-marzo 2015: 143-156.

\section{Semblanzas de los autores}

Alicia Esther Quintana: Licenciada en matemática y especialista en gestión de la calidad por la Universidad Nacional del Sur (UNS), Argentina. Profesora de estadística e investigadora de la UNS, categorizada por el Sistema Nacional. Su área de especialización es control de calidad, con énfasis en cartas de control estadístico de procesos. Ha publicado trabajos en revistas y reuniones científicas y participó en proyectos de investigación con esa temática. Actualmente participa en un proyecto sobre herramientas de mejora en la metodología Six Sigma. Realizó asesoramientos estadísticos a empresas del medio y fue miembro de proyectos educativos a distancia en calidad en la UNS.

María Virginia Pisani: Licenciada en economía por la Universidad Nacional del Sur (UNS), Argentina. Aspirante al grado de doctor en ciencias de la administración por la UNS. Fue docente del Departamento de Economía, UNS. Es docente del Departamento de Matemática de la UNS en el área probabilidad y estadística y de la Universidad Provincial del Sudoeste (UPSO) en las asignaturas economía y estadística. Trabaja en proyectos de investigación relacionados con la metodología Six Sigma y la economía de la salud. Ha publicado trabajos en revistas y anales de reuniones científicas en estas temáticas.

Casal Ricardo Néstor: Ingeniero industrial por la Universidad Nacional del Sur (UNS), Argentina. Máster en estadística matemática y experto en economía industrial. Es docente investigador categorizado por el Sistema Nacional y profesor titular del Departamento de Ingeniería, UNS, en las asignaturas modelización y métodos de la ingeniería industrial y gestión de calidad. Dirige proyectos de investigación en temas de calidad y planificación, así como control de la producción en la UNS. Dirigió tesis de magister y doctorado, ha impartido cursos de postgrado y ha publicado numerosos trabajos en revistas y anales de congresos nacionales e internacionales. Fue director decano del departamento de ingeniería, UNS, en 4 oportunidades y secretario de investigación del mismo departamento. 